\title{
Reverted Mean and Asymptotic Stationary Distribution for Timber Price Using a Mean-Reverting Stochastic Model
}

\author{
Yoshimoto, A. ${ }^{* 1} \&$ Jimenez, J.C. ${ }^{* 1 * 2}$ \\ Keywords: asymptotic distribution, market log price, mean-reverting process, \\ stochastic differential equation, stochastic model \\ Abstract: We use a mean-reverting stochastic model to demonstrate a pro- \\ cedure for calculating the reverted mean and asymptotic stationary \\ distribution of sawlog price data. The data used for this study comes \\ from a local auction market in the Fukuoka Prefecture, Japan where \\ $4 \mathrm{~m}$ long sugi (Cryptomeria japonica) and hinoki (Chamercypress \\ obtusa) are commonly traded. Parameter estimation is completed \\ using a quasi-maximum likelihood method based on a local lineariza- \\ tion scheme. The stationary distribution for our stochastic model is \\ numerically constructed using a generalized Pearson system.
}

Received January 22, 2012; Accepted February 14, 2012

${ }^{* 1}$ Institute of Statistical Mathematics, Japan

*2 Instituto de Cibernética, Matemática y Física, Cuba 


\section{Introduction}

Many researchers have acknowledged the importance of considering the stochastic nature of management decisions. Among them are Reed (1984), Brazee and Mendelsohn (1988), Clarke and Reed (1989), Clarke and Reed (1990), Teeter and Caulfield (1991), Zinkhan (1991), Thomson (1992), Reed and Ye (1994), Yin and Newman (1995), Yin and Newman (1996), Yoshimoto and Shoji (1998), Willassen (1998), Plantinga (1998), Morck et al. (1989), Thorsen (1999), Brazee and Bulte (2000), Fina et al. (2000), Hughes (2000), Yoshimoto (2002), Sødal (2003), Insley (2002), Insley and Rollins (2005) and Penttinen (2006). Geometric Brownian motion is widely used in stochastic modeling because it is easily applied in this context. Clarke and Reed (1989), Thomson (1992), Yoshimoto and Shoji (1998), and Yoshimoto (2002) have supported the use of geometric Brownian motion for price dynamics, whereas Haight and Holmes (1991) applied a non-stationary random walk to a log-transformed price process.

Recognizing that the long-run marginal cost of production reflects the mean market price (a microeconomic concept), Insley (2002) and Insley and Rollins (2005) employed a mean-reverting process in their models. Yoshimoto (2008) employed four variants of a mean-reverting stochastic model to analyze reverted mean price using local market data from 1970 to 2006, as well as national monthly averages. Yoshimoto (2009) constructed a stochastic dynamic programming model that applied mean-reverting processes to seek the minimum harvest age and threshold price for sustainable forest management.

The reverted mean price plays an important role in making decisions about future management activities. Likewise, information about the future variance or distribution of price dynamics is needed when analyzing the risk that forest owners will abandon active management. The objective of this paper is to demonstrate use of a mean-reverting 
stochastic model to calculate the reverted mean and asymptotic stationary distribution of sawlog price data. We use market-based price data for $4 \mathrm{~m}$ sugi (Cryptomeria japonica) and hinoki (Chamercypress obtusa) sawlogs taken from the Fukuoka Prefecture, Japan. The remainder of this paper is organized as follows. In the next section we describe the mean-reverting stochastic model and parameter estimation method. In the third section, we derive the reverted mean and asymptotic stationary distribution of price dynamics of our model. Whereas in the fourth section we compute the reverted mean and asymptotic stationary distribution of the model estimated from the data. The final section presents some concluding remarks.

\section{Mean-Reverting Processes and Parameter Estimation}

In this paper, we use the following mean-reverting stochastic model for log price dynamics to estimate the reverted mean of the price over time and derive its asymptotic stationary distribution:

[1] $\quad d x_{t}=\left(\alpha-\beta x_{t}\right) d t+x_{t} \sigma d B_{t}$

where $x_{t}$ is the $\log$ price at time $t$, and $B_{t}$ is a standard Brownian motion with the following characteristics:

1. $B_{0}=0$

2. $\left\{B_{t}, t \geq 0\right\}$ has independent increments with Gaussian distribution $B_{t}-B_{s} \sim N(0, t-s)$

The set of parameters $(\alpha, \beta, \sigma)$ are assumed to be positive so that equation is strictly constrained to be mean-reverting.

Parameter estimation for the model presented above is achieved via a pseudo-likelihood approach based on a local linearization method (Ozaki, 1985a, 1992, 1993, Shoji and Ozaki, 1997, and Shoji, 1998). A number of comparative studies among different estimation methods 
have shown that in practical situations the pseudo-likelihood estimators based on the local linearization method outperform other for simplicity, computational efficiency and negligible bias (see Shoji and Ozaki ,1997, Durham and Gallant, 2002, Singer 2002, Hurn et al., 2007). In brief, the method begins by converting a nonlinear stochastic differential equation into a stochastic differential equation with a constant volatility term. Then, over a small time lapse, the nonlinear drift term of the stochastic differential equation is locally approximated by a linear function of the state and time. The resulting linear stochastic differential equation can be solved analytically, which enables derivation of the corresponding likelihood function for parameter estimation. A detailed description of the method can be found in Yoshimoto and Shoji (2002).

Following Yoshimoto and Shoji (2002), the quasi log-likelihood function for the data set, $\left\{x_{t}\right\}$, is derived as

[2] $\quad-\frac{1}{2} \sum_{i=1}^{N}\left\{\frac{\left(y_{t_{i}}-E_{t_{i-1}}\right)^{2}}{V_{t_{i-1}}}+\log \left(2 \pi V_{t_{i-1}}\right)\right\}+\log \left(p\left(y_{t_{0}}\right)\right)$

where

$$
\begin{array}{ll}
{[3]} & E_{t_{i}}=y_{t_{i}}+\frac{h\left(y_{t_{i}}\right)}{L_{t_{i}}}\left(e^{L_{t_{i}} \Delta t}-1\right)+\frac{M_{t_{i}}}{L_{t_{i}}^{2}}\left(e^{L_{t_{i}} \Delta t}-1-L_{t_{i}} \Delta t\right) \\
{[4]} & V_{t_{i}}=\frac{\sigma^{2} \cdot\left(\exp \left(2 L_{t_{i}} \Delta t\right)-1\right)}{2 L_{t_{i}}} \\
{[5]} & y_{t_{i}}=\phi\left(x_{t_{i}}\right)=\ln \left(x_{t_{i}}\right) \\
{[6]} & h\left(y_{t_{i}}\right)=\frac{1}{x_{t_{i}}} \cdot\left(\alpha-\beta x_{t_{i}}\right)-\frac{1}{2} \sigma^{2} \\
{[7]} & L_{t_{i}}=-\frac{\alpha}{x_{t_{i}}} \\
{[8]} & M_{t_{i}}=\frac{\alpha \sigma^{2}}{2 x_{t_{i}}}
\end{array}
$$

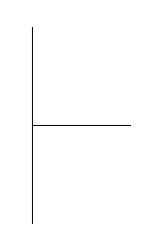


Note that $t_{i}$ is the time of the $i^{\text {th }}$ observation and $y_{t_{i}}$ is the corresponding log-transformed data of $x_{t_{i}}$. To strictly constrain parameters $(\alpha, \beta, \sigma)$ to positive values, the following exponential transformation is applied

$[9]$

$$
\alpha=e^{\theta_{1}}, \beta=e^{\theta_{2}}, \sigma=e^{\theta_{3}}
$$

where the new parameters $\left(\theta_{1}, \theta_{2}, \theta_{3}\right)$ are unrestricted in the range of $(-\infty, \infty)$. All parameters are estimated by maximizing the above log-likelihood function.

\section{Reverted Mean Values and Asymptotic Stationary Distri- bution}

The reverted mean is derived by first converting the stochastic differential equation into a form that includes a constant volatility term, using the following transformation:

$[10] \quad y_{t}=\ln \left(x_{t}\right)$

With this conversion, we have the following constant volatility process:

$$
d y_{t}=\left\{\alpha e^{-y_{t}}-\beta-\frac{1}{2} \sigma^{2}\right\} d t+\sigma d B_{t}
$$

Setting the drift term equal to zero, we get the reverted mean, $x_{r}$, as a function of all parameter estimates:

$[12] \quad x_{r}=\frac{2 \alpha}{2 \beta+\sigma^{2}}$

To derive the asymptotic stationary distribution, we first consider the transition density function, $P$, of the diffusion process, which is governed by the following general form of a stochastic differential equation:

$$
d x=f(x) d t+g(x) d w
$$
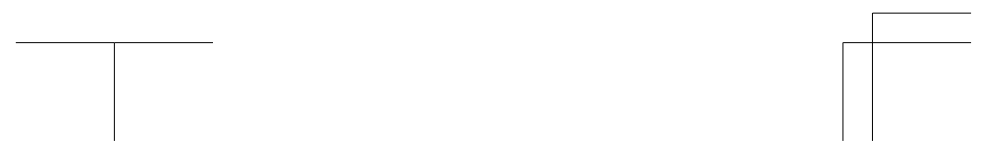
with $x\left(t_{0}\right)=x_{0}$. This density function $P$ satisfies the so-called Kolmogorov Forward Equation (KFE)

$$
\frac{d P(t, x)}{d t}=-\frac{\partial}{\partial x}(f(x) P(t, x))+\frac{1}{2} \frac{\partial^{2}}{\partial x^{2}}\left(g^{2}(x) P(t, x)\right)
$$

for all $t>t_{0}$. When $t$ goes to infinity, the stationary distribution $P_{\infty}$ of the diffusion process $x$ is then defined by the following generalized Pearson system:

$$
\frac{d P_{\infty}(x)}{d x}=\frac{2 f(x)-g(x) g^{\prime}(x)}{g^{2}(x)} P_{\infty}(x)
$$

which results in the KFE by setting $\frac{d P(t, x)}{d t}=0$ (see Ozaki, 1985b). Thus, for the mean-reverting stochastic model used here, the stationary distribution of $x$ is governed by

[16] $\quad \frac{d P_{\infty}(x)}{d x}=\frac{2(\alpha-\beta x)-\sigma^{2} x}{\sigma^{2} x^{2}} P_{\infty}(x)$

This equation is numerically integrated using a classical order-2 local linearization method for ordinary differential equations (Jimenez et al., 2005), defined by the recursive formula

$$
P_{n+1}=P_{n}+L e^{A_{n} h_{n}} r
$$

where $P_{n}=P_{\infty}\left(x_{n}\right)$ and $x_{n}=n h_{n}$ for all $n=0, \ldots, N$, and $P_{0}=0$. Here, $N$ denotes the maximum number of iterations, and $h_{n}$ the stepsize at each iteration $n+1$.

To compute $P_{n}$ in our example, we set $N=10^{7}, h_{n}=0.01$ for all $n$, and the matrices

$$
\begin{aligned}
& A_{n}=\left[\begin{array}{ccc}
F\left(x_{n}\right) & \frac{d}{d x_{n}} F\left(x_{n}\right) P_{n} & F\left(x_{n}\right) P_{n} \\
0 & 0 & 1 \\
0 & 0 & 0
\end{array}\right], \\
& L=\left[\begin{array}{lll}
1 & 0 & 0
\end{array}\right]
\end{aligned}
$$
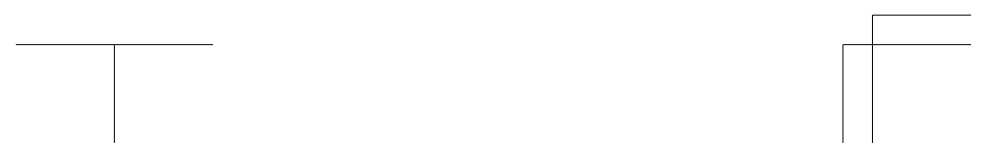
and

$$
r=\left[\begin{array}{l}
0 \\
0 \\
1
\end{array}\right]
$$

with

$$
F(x)=\frac{2(\alpha-\beta x)-\sigma^{2} x}{\sigma^{2} x^{2}}
$$

The normalized density function $p_{\infty}$ is finally computed as

$$
\text { [18] } \quad p_{\infty}\left(x_{i}\right)=\frac{P_{\infty}\left(x_{i}\right)}{\sum_{i=0}^{N} P_{\infty}\left(x_{i}\right) h_{i}}
$$

The mean $M$, variance $S t d^{2}$, skewness $S k w$, and kurtosis $K u r$ of $p_{\infty}$ are defined as follows:

$$
\begin{aligned}
& M=\sum_{i=0}^{N} x_{i} p_{\infty}\left(x_{i}\right) h_{i} \\
& S t d^{2}=\sum_{i=0}^{N}\left(x_{i}-M\right)^{2} p_{\infty}\left(x_{i}\right) h_{i} \\
& S k w=\frac{1}{S t d^{3}} \sum_{i=0}^{N}\left(x_{i}-M\right)^{3} p_{\infty}\left(x_{i}\right) h_{i} \\
& \text { Kur }=\frac{1}{S t d^{4}} \sum_{i=0}^{N}\left(x_{i}-M\right)^{4} p_{\infty}\left(x_{i}\right) h_{i}
\end{aligned}
$$

\section{Demonstrative Examples}

For demonstrative purposes, we use time series sawlog price data for two different species, sugi (Cryptomeria japonica) and hinoki (Chamaecypress obtusa). This market-based data, which includes 1,075 sugi and 1,074 hinoki data points, was taken from Fukuoka prefecture log market (Ukiha log auction). The sawlogs were $4 \mathrm{~m}$ in length and diameter 
ranged from $14-18 \mathrm{~cm}$. Figure 1 shows the price dynamics of these data sets from April 7, 1970 to December 18, 2009.
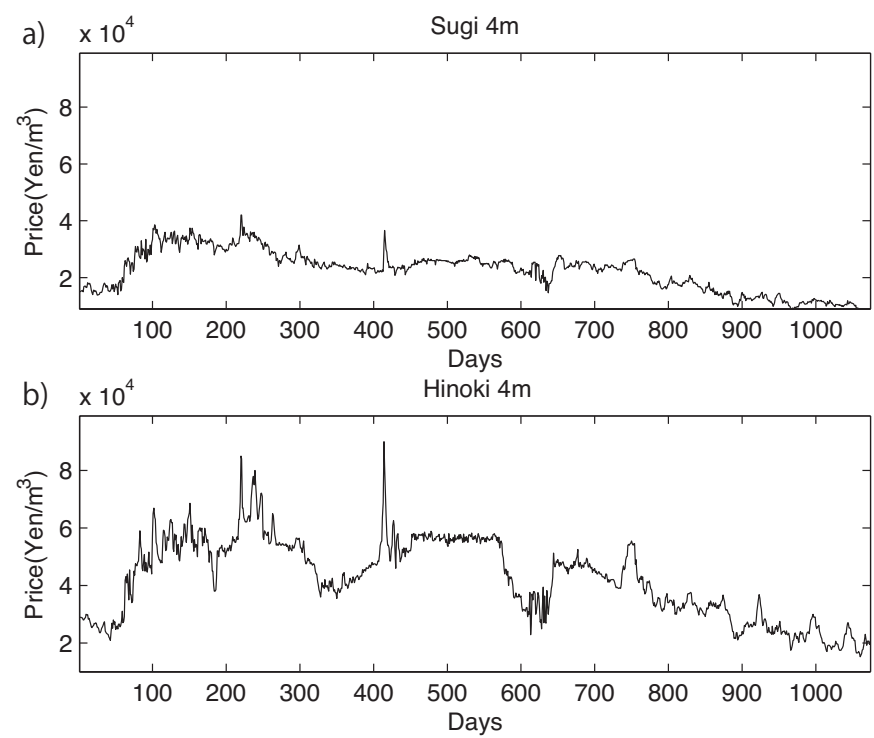

Figure 1. Price dynamics of $4 \mathrm{~m}$ sawlogs.

a) sugi (Cryptomeria japonica), b) hinoki (Chamaecypress obtusa)

Estimated parameter values, the derived reverted mean, and statistics for the asymptotic stationary distribution of the two data sets are provided in Table 1. Comparing the estimates between sugi and hinoki in terms of the value of $\sigma$, the price of sugi showed slightly less volatility than that of hinoki. From Eq.[12], the reverted mean of the price for sugi was $17,413 \mathrm{Yen} / \mathrm{m}^{3}$, while it was $35,321 \mathrm{Yen} / \mathrm{m}^{3}$ for hinoki. Note that the mean of the asymptotic stationary distribution is not the same as the reverted mean because the distribution is not Gaussian. Thus, the most likely value or mode differs from the mean of the distribution (Figure 2). 
Table 1. Parameter estimates, reverted mean, and asymptotic stationary distribution.

\begin{tabular}{llll}
\hline \multirow{3}{*}{ Parameter } & \multicolumn{1}{c}{ Sugi } & Hinoki \\
\cline { 2 - 4 } & $\beta$ & 3029.345 & 10688.812 \\
\cline { 2 - 4 } & $\beta$ & 0.132952 & 0.2524661 \\
\hline Reverted Mean* & $x_{r}$ & 0.082041 & 0.1003003 \\
\hline \multirow{2}{*}{$\begin{array}{l}\text { Asymptotic } \\
\text { Stationary } \\
\text { Distribution }\end{array}$} & Mean $M$ & 17,413 & 35,321 \\
\cline { 2 - 4 } & Standard Dev Std & 29,840 & 47,865 \\
\cline { 2 - 4 } & Skewness Skw & 5.7025 & $27,482.69$ \\
\cline { 2 - 4 } & Kurtosis $K u r$ & 1.5103 & 3.8273 \\
\hline *
\end{tabular}

*Note: The reverted mean is also the distribution's mode, or most frequently observed value.

\section{Conclusions}

In this paper we used a mean-reverting stochastic model to demonstrate a procedure for deriving the reverted mean price and asymptotic stationary distribution for sawlog prices. The reverted mean was derived by first converting the stochastic differential equation into a form that includes a constant volatility term, then setting the drift term equal to zero. The asymptotic stationary distribution was derived in terms of the general Pearson system, which was numerically integrated with the classical order-2 local linearization method.

Our analysis showed that the reverted mean price for sugi sawlogs was much less than that for hinoki. On the other hand, the variance of the asymptotic stationary distribution for sugi was less than that for hinoki, suggesting sugi sawlog prices may be more stable than hinoki prices (based on the market data used for this analysis). Many researchers have suggested use of a stochastic dynamic programming model to identify the minimum threshold price level at which forest owners will maintain management practices. Using this information in 


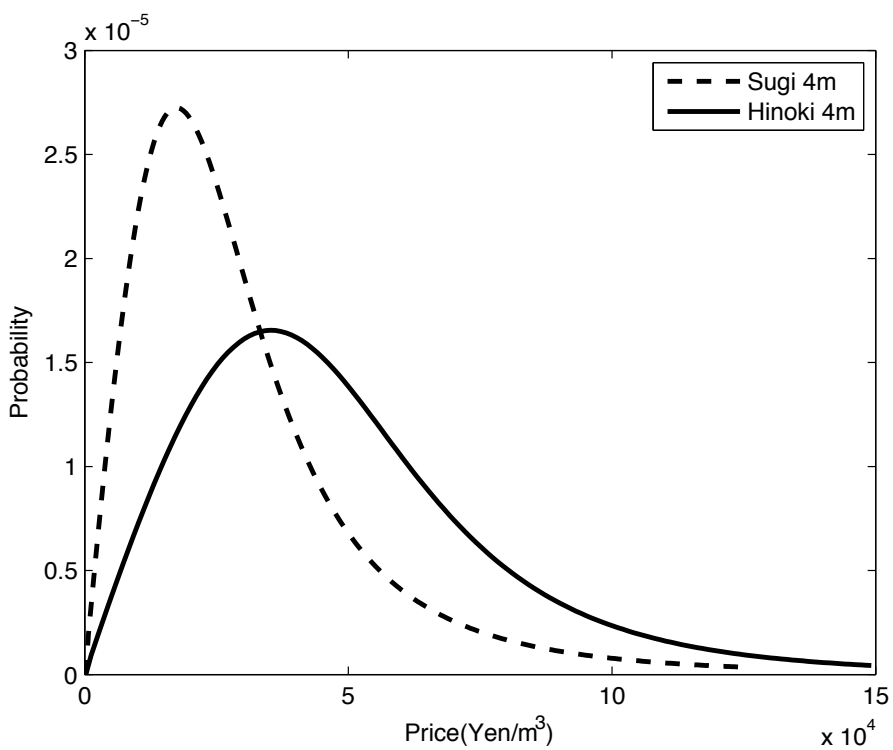

Figure 2. Asymptotic stationary distribution.

conjunction with the asymptotic stationary distribution of price demonstrated here, it is possible to estimate the level of risk or probability that forest owners will abandon active management. If sustainable forest management is desired in a certain area, it may be possible to issue an insurance policy that is based on this derived probability to avoid the abandonment of management practices.

\section{References}

Brazee, R. and Bulte, E. (2000) Optimal harvesting and thinning with stochastic prices, Forest Sci. 46: 23-31.

Brazee, R. and Mendelsohn, R. (1988) Timber harvesting with fluctuating prices, Forest Sci. 34: 359-372. 
Clarke, H.R. and Reed, W.J. (1989) The tree-cutting problem in a stochastic environment: The case of age-dependent growth, J. Econ. Dyn. Control 13: 569-595.

Clarke, H.R. and Reed, W.J. (1990) Land development and wilderness conservation policies under uncertainty: a synthesis, Nat. Resour. Model. 4: 11-37.

Durham, G.B. and Gallant, A.R. (2002) Numerical techniques for maximum likelihood estimation of continuous-time diffusion processes, J. Bus. Econ. Stat. 20: 297-316.

Fina, M., Amache,r G.S. and Sullivan, J. (2000) Uncertainty, debt, and forest harvesting: Faustmann revisited, Forest Sci. 47: 188-196.

Haight, R.G. and Holmes, T.P. (1991) Stochastic price models and optimal tree cutting: Results for loblolly pine, Nat. Resour. Model. 5: $423-443$.

Hughes, W.R. (2000) Valuing a forest as a call option: The sale of forestry corporation of New Zealand, Forest Sci. 46: 32-39.

Hurn, A.S., Jeisman, I.J. and Lindsay, K.A. (2007) Seeing the wood for the trees: a critical evaluation of methods to estimate the parameters of stochastic differential equations, J. Financ. Economet. 5: $390-455$.

Insley, M. (2002) A real options approach to the valuation of a forestry investment, J. Environ. Econ. Manag. 44: 471-492.

Insley, M. and Rollins, K. (2005) On solving the multirotational timber harvesting problem with stochastic prices: a linear complementary formulation, Am. J. Agr. Econ. 87: 735-755.

Jimenez, J.C. and Carbonell, F. (2005) Rate of convergence of local linearization schemes for initial-value problems, Appl. Math. Comput. 171: $1282-1295$.
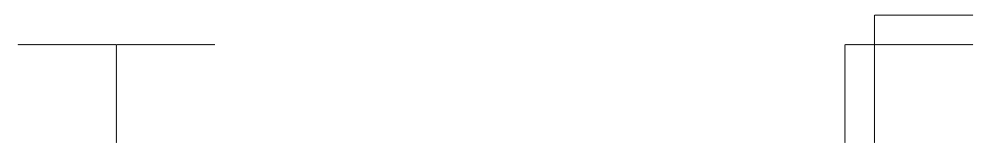
Morck, R., Schwartz, E. and Stangeland, D. (1989) The valuation of forestry resources under stochastic prices and investments, J. Financ. Quant. Anal. 24: 473-487.

Ozaki, T. (1985a) Statistical identification of storage models with application to stochastic hydrology, J. Am. Water Resour. Assoc. 21: 663-675.

Ozaki, T. (1985b) Nonlinear time series models and dynamical systems, In: Hannan E.J. et al. (Eds.) Handbook of Statistics. Vol 5, NorthHolland, p.25-83.

Ozaki, T. (1992) A bridge between nonlinear time series models and nonlinear stochastic dynamical systems: a local linearization approach, Stat. Sinica 2: 113-135.

Ozaki, T. (1993) A local linearization approach to nonlinear filtering, Int. J. Control 57: 75-96.

Pettinen, M.J. (2006) Impact of stochastic price and growth processes on optimal rotation age, Eur. J. Forest Res. 125: 335-343.

Plantinga, A.J. (1998) The optimal timber rotation: An option value approach, Forest Sci. 44: 192-202.

Reed, W.J. (1984) The effects of the risk of fire on the optimal rotation of a forest, J. Environ. Econ. Manag. 11: 180-190.

Reed, W.J. and Ye, J.J. (1994) The role of stochastic monotonicity in the decision to conserve or harvest old-growth forest, Nat. Resour. Model. 8: 47-9.

Shoji, I. (1998) Approximation of continuous time stochastic processes by a local linearization method, Math. Comput. 67: 287-298.

Shoji, I. and Ozaki, T. (1997) Comparative study of estimation methods for continuous time stochastic processes, J. Time Ser. Anal. 18: $485-506$. 
Singer, H. (2002) Parameter estimation of nonlinear stochastic differential equations: Simulated maximum likelihood versus extended Kalman filter and Ito-Taylor expansion, J. Comput. Graph. Stat. 11: $972-995$.

Sødal, S. (2002) The stochastic rotation problem: A comment, J. Econ. Dyn. Control 26: 509-515.

Teeter, L.D. and Caulfield, J.P. (1991) Stand density management strategies under risk: Effects of stochastic prices, Can. J. Forest Res. 21: 1373-1379.

Thomson, T.A. (1992) Optimal forest rotation when stumpage prices follow a diffusion process, Land Econ. 68: 329-342.

Thorsen, B.J. (1999) Afforestation as a real option: Some policy implications, Forest Sci. 45: 171-178.

Willassen, Y. (1998) The stochastic rotation problem: A generalization of Faustmann's formula to stochastic forest growth, J. Econ. Dyn. Control 22: 573-596.

Yin, R. and Newman, D. (1995) A note on the tree-cutting problem in a stochastic environment, J. Forest Econ. 1: 181-190.

Yin, R. and Newman, D. (1996) The effect of catastrophic risk on forest investment decisions, J. Environ. Econ. Manag. 31: 186-197.

Yoshimoto, A. (2002) Stochastic control modeling for forest stand management under uncertain price dynamics through geometric Brownian motion, J. Forest Res. 7: 81-90.

Yoshimoto, A. (2008) Analysis on the reverted mean of log price dynamics through stochastic modeling, FORMATH 7: 61-77.

Yoshimoto, A. (2009) Threshold price as an economic indicator for sustainable forest management under stochastic log price, J. Forest Res. 14: 193-202.
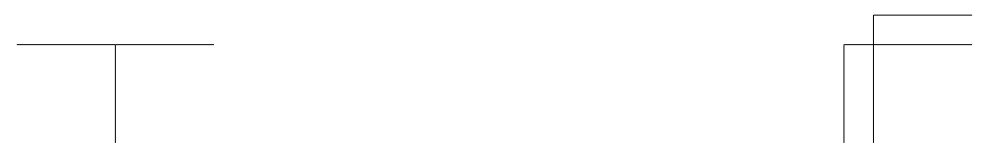
Yoshimoto, A. and Shoji, I. (1998) Searching for an optimal rotation age for forest stand management under stochastic log prices, Eur. J. Oper. Res. 105: 100-112.

Yoshimoto, A. and Shoji, I. (2002) Comparative analysis of stochastic models for financial uncertainty in forest management, Forest Sci. 48: $755-766$.

Zinkhan, F.C. (1991) Option pricing and timberland's land-use conversion option, Land Econ. 67: 317-325. 